\title{
Tuning the outward to inward swelling in lithiated silicon nanotubes via surface oxide coating
}

\author{
Jiangwei Wang ${ }^{1,2, \#, *}$, Hao Luo ${ }^{3, \#}$, Yang Liu ${ }^{4, \#, ~ Y a n g ~ H e}{ }^{2}$, Feifei Fan ${ }^{5}$, Ze Zhang ${ }^{1}$, \\ Scott X. Mao ${ }^{2, *}$, Chongmin Wang ${ }^{6, *}$ and Ting $\mathrm{Zhu}^{3}$
}

${ }^{1}$ Center of Electron Microscopy and State Key Laboratory of Silicon Materials, School of Materials Science and Engineering, Zhejiang University, Hangzhou 310027 China

${ }^{2}$ Department of Mechanical Engineering and Materials Science, University of Pittsburgh, Pittsburgh, Pennsylvania, 15261, United States

${ }^{3}$ Woodruff School of Mechanical Engineering, Georgia Institute of Technology, Atlanta, Georgia, 30332, United States

${ }^{4}$ Department of Materials Science and Engineering, North Carolina State University, Raleigh, North Carolina, 27695, United States

${ }^{5}$ Department of Mechanical Engineering, University of Nevada, Reno, Nevada 89557, United States

${ }^{6}$ Environmental Molecular Sciences Laboratory, Pacific Northwest National Laboratory, Richland, Washington 99352, United States

Corresponding Authors: C. W. (chongmin.wang@pnnl.gov); J.W. (iiangwei_wang@zju.edu.cn); S.X.M. (sxm2@pitt.edu). 


\section{Methods}

\section{Sample preparation and in situ lithiation experiment}

The $a$-Si nanotubes were obtained via the following steps. The $a$-Si/carbon nanofiber $(a$ $\mathrm{Si} / \mathrm{CNF}$ ) nanocomposites were prepared by coating $a$-Si onto CNFs using a chemical vapor deposition (CVD) method (Applied Sciences Inc.) ${ }^{1,2}$. The thickness of $a$-Si layers were about $\sim 20-25 \mathrm{~nm}$ after 30 min coating. Then the $a$-Si/CNF nanocomposites were heated in air at $500^{\circ} \mathrm{C}$ for $2 \mathrm{~h}$ to remove the CNF core. TEM observations showed that the CNF had been completed removed, and the STEM line-scan analysis indicated that a negligible native $\mathrm{SiO}_{\mathrm{x}}$ layer (less than $2 \mathrm{~nm}$ ) formed on both the inner and outer surfaces of $a$-Si nanotubes, due to the low thermal treatment temperature and short treatment time ${ }^{3}$. To obtain thicker $\mathrm{SiO}_{\mathrm{x}}$ layers, the $a$-Si nanotubes were further oxidized for longer time in air. The $3\|4 \mathrm{~nm}, 4\| 6.5 \mathrm{~nm}$ and $6.5 \| 9 \mathrm{~nm} \mathrm{SiO} / \mathrm{Si} / \mathrm{SiO}_{\mathrm{x}}$ nanotubes were fabricated by oxidization of $a$-Si nanotubes with native oxides at $500^{\circ} \mathrm{C}$ for 1 hour, 4 hours and 6 hours, respectively. The in situ electrochemical experiment was conducted inside TEM (FEI Titan 80-300 TEM with imaging lens spherical aberration corrector, operated at $300 \mathrm{kV}$ ) by using a nanoscale battery, which consists of a working electrode of an individual $a$-Si nanotube, a counter-electrode of bulk Li metal and a solid electrolyte of naturally-grown lithium oxide $\left(\mathrm{Li}_{2} \mathrm{O}\right)$. The detailed setup and procedure of the in situ TEM experiments have been documented in our previous publications ${ }^{2,4,5}$. In Figure 1 and Figure S3-5, the diameter-time curves were measured at a given position of nanotubes and the different lithiation stages in all samples were identified based on the change of lithiation geometries in real-time observations, i.e. the first-stage lithiation finished once the sandwich structure disappeared and then the lithiation entered into the second-stage. 
Besides, under low-magnification, the wall thickness and surface oxides of $a$-Si nanotubes cannot be clearly identified during the lithiation (Figure S7). Since the main purpose of this work is to clearly understand the lithiation behavior and lithiation dynamics of $a$-Si nanotubes with different surface $\mathrm{SiO}_{\mathrm{x}}$ layers, we only presented in the paper the real-time high-magnification images (with the nanometer resolution), by which the geometrical changes during lithiation can be precisely measured.

\section{Chemomechanical model}

We adapted a chemomechanical model from our previous work ${ }^{4}$ to simulate the twostage lithiation process in an $a$-Si nanotube, involving the sandwich, two-phase lithiation during the first stage and the single-phase lithiation during the second stage. To simulate the two-phase lithiation, we solved the non-linear diffusion equation with the $\mathrm{Li}$ diffusivity depending on local Li concentration ${ }^{6,7}$. The sandwich process was enabled by prescribing a saturated $\mathrm{Li}$ concentration (corresponding to the product phase of $a-\mathrm{Li}_{2.3} \mathrm{Si}$ ) as the boundary condition at both the outer and inner surfaces of the nanotube, given the fact that Li diffusion is much faster on the surface than in the bulk of Si. Then we also simulated the second stage of single-phase lithiation by tuning the Li concentrationdependent diffusivity ${ }^{7}$. To model the lithiation-induced deformation, we employed a rate formulation for a chemo-elastic-plastic constitutive law ${ }^{7}$, which is necessary to characterize large strains involved during lithiation. In this formulation, the total strain

rate $\dot{\varepsilon}_{i j}$ is taken to be the sum of three contributions, $\dot{\varepsilon}_{i j}=\dot{\varepsilon}_{i j}^{c}+\dot{\varepsilon}_{i j}^{e}+\dot{\varepsilon}_{i j}^{p}$. Here $\dot{\varepsilon}_{i j}^{c}$ is the rate of the lithiation-induced chemical strain; it is proportional to the rate of the normalized $\mathrm{Li}$ 
concentration $\dot{c}$ according to $\dot{\varepsilon}_{i j}^{c}=\beta_{i j} \dot{c}$, where $\beta_{i j}$ is the lithiation expansion coefficient and $c$ varies between 0 and 1 (e.g., from $a-\mathrm{Si}$ to $\mathrm{Li}_{2.3} \mathrm{Si}$ during the first stage of lithiation; and from $\mathrm{Li}_{2.3} \mathrm{Si}_{1}$ to $\mathrm{Li}_{15} \mathrm{Si}_{4}$ in the second stage); $\dot{\varepsilon}_{i j}^{e}$ is the elastic strain rate; and $\dot{\mathcal{E}}_{i j}^{p}$ is the plastic strain rate.

To numerically implement this chemomechanical model ${ }^{4}$, the coefficients of anisotropic lithiation expansion $\beta_{i j}$ need to be determined. In the case of the $a-\mathrm{Si}$ nanotube with native oxides, the experimentally measured volume expansions of $\left(V_{1}-V_{0}\right) / V_{0}$ and $\left(V_{2}-V_{0}\right) / V_{0}$ at the end of the first-stage and second-stage lithiation were respectively about $172 \%$ and $252 \%$, where $V_{0}$ denotes the initial volume of $a$-Si, and $V_{1}$ the volume after the first-stage lithiation and $V_{2}$ the volume after the second-stage lithiation. The corresponding logarithmic volume strains were respectively calculated as $\epsilon_{V 1}=\log \left(\frac{V_{1}}{V_{0}}\right)=1.00$ and $\epsilon_{V 2}=\log \left(\frac{V_{2}}{V_{1}}\right)=0.26$. We assumed all the chemical strains occurred in the cross section of the nanotube. For the first-stage lithiation, we assigned the coefficients of anisotropic lithiation expansion in the radial and hoop directions as $\beta_{r}=0.93$ and $\beta_{\theta}=0.07$, so that $\beta_{r}+\beta_{\theta}=\epsilon_{V 1}$; other components of $\beta_{i j}$ were zero. For the second-stage lithiation, we took $\beta_{r}=0.24$ and $\beta_{\theta}=0.02$, so that $\beta_{r}+\beta_{\theta}=\epsilon_{V 2}$. The same set of parameters were used in our chemomechanical modeling of $6.5 \| 9 \mathrm{~nm}$ $\mathrm{SiO}_{\mathrm{x}} / \mathrm{Si} / \mathrm{SiO}_{\mathrm{x}}$ nanotube. Additionally, the experimentally measured volume expansion of the lithiated $\mathrm{SiO}_{\mathrm{x}}$ layer was about $82 \%$, corresponding to a logarithmic volume strain of about 0.60. We took $\beta_{r}=0.51$ and $\beta_{\theta}=0.09$ for the lithiated $\mathrm{SiO}_{\mathrm{x}}$ layer. Young's modulus for $a$-Si and fully lithiated $a-\mathrm{Si}$ is $100 \mathrm{GPa}$ and $40 \mathrm{GPa}$ respectively; the corresponding yield stress is $5 \mathrm{GPa}$ and $1 \mathrm{GPa}$; Young's modulus and the yield stress of 
partially lithiated $a-\mathrm{Si}$ is a linear interpolation between $a$-Si and fully lithiated $a$-Si;

Poisson's ratio is 0.22 for all the phases. For the surface oxide $\mathrm{SiO}_{\mathrm{x}}$ and its lithiated product, Young's modulus is $170 \mathrm{GPa}$, the yield stress is $5 \mathrm{GPa}$, and Poisson's ratio is 0.17 .

Figure S6 shows the model setup that incorporates the oxide layer at both the inner and outer surfaces of the $6.5 \| 9 \mathrm{~nm} \mathrm{SiO} \times{ }_{x} / \mathrm{Si}_{/} / \mathrm{SiO}_{\mathrm{x}}$ nanotube. This model accounted for the sandwich lithiation mechanism and two-stage lithiation process, as well as the lithiation of $\mathrm{SiO}_{\mathrm{x}}$ at both the outer and inner surfaces. The same set of anisotropic chemical strains as the earlier case of native oxides was used. This indicates that our chemical strain coefficients captured the dominant effects of anisotropic expansion, irrespective of the thickness of surface oxides. In addition, we used the experimentally measured value of lithiation-induced volume expansion in $\mathrm{SiO}_{\mathrm{x}}(\sim 82 \%)$, which is much smaller than that of $a$-Si $(\sim 270 \%)$.

\section{Reference:}

1 Wang, C.-M., Li, X., Wang, Z., Xu, W., Liu, J., Gao, F., Kovarik, L., Zhang, J.-G., Howe, J., Burton, D. J., Liu, Z., Xiao, X., Thevuthasan, S. \& Baer, D. R. Nano Lett. (2012), 12, 1624-1632.

2 Wang, J. W., Liu, X. H., Zhao, K., Palmer, A., Patten, E., Burton, D., Mao, S. X., Suo, Z. \& Huang, J. Y. ACS Nano (2012), 6, 9158-9167.

3 Karki, K., Zhu, Y., Liu, Y., Sun, C.-F., Hu, L., Wang, Y., Wang, C. \& Cumings, J. ACS Nano (2013), 7, 8295-8302.

4 Wang, J. W., He, Y., Fan, F., Liu, X. H., Xia, S., Liu, Y., Harris, C. T., Li, H., Huang, J. Y., Mao, S. X. \& Zhu, T. Nano Lett. (2013), 13, 709-715.

5 Liu, X. H., Wang, J. W., Huang, S., Fan, F., Huang, X., Liu, Y., Krylyuk, S., Yoo, J., Dayeh, S. A. \& Davydov, A. V. Nat. Nanotechnol. (2012), 7, 749-756.

6 Liu, X. H., Zheng, H., Zhong, L., Huang, S., Karki, K., Zhang, L. Q., Liu, Y., Kushima, A., Liang, W. T., Wang, J. W., Cho, J.-H., Epstein, E., Dayeh, S. A., Picraux, S. T., Zhu, T., Li, J., Sullivan, J. P., Cumings, J., Wang, C., Mao, S. X., Ye, Z. Z., Zhang, S. \& Huang, J. Y. Nano Lett. (2011), 11, 3312-3318.

7 Huang, S., Fan, F., Li, J., Zhang, S. L. \& Zhu, T. Acta Materialia (2013), 61, 4354-4364. 

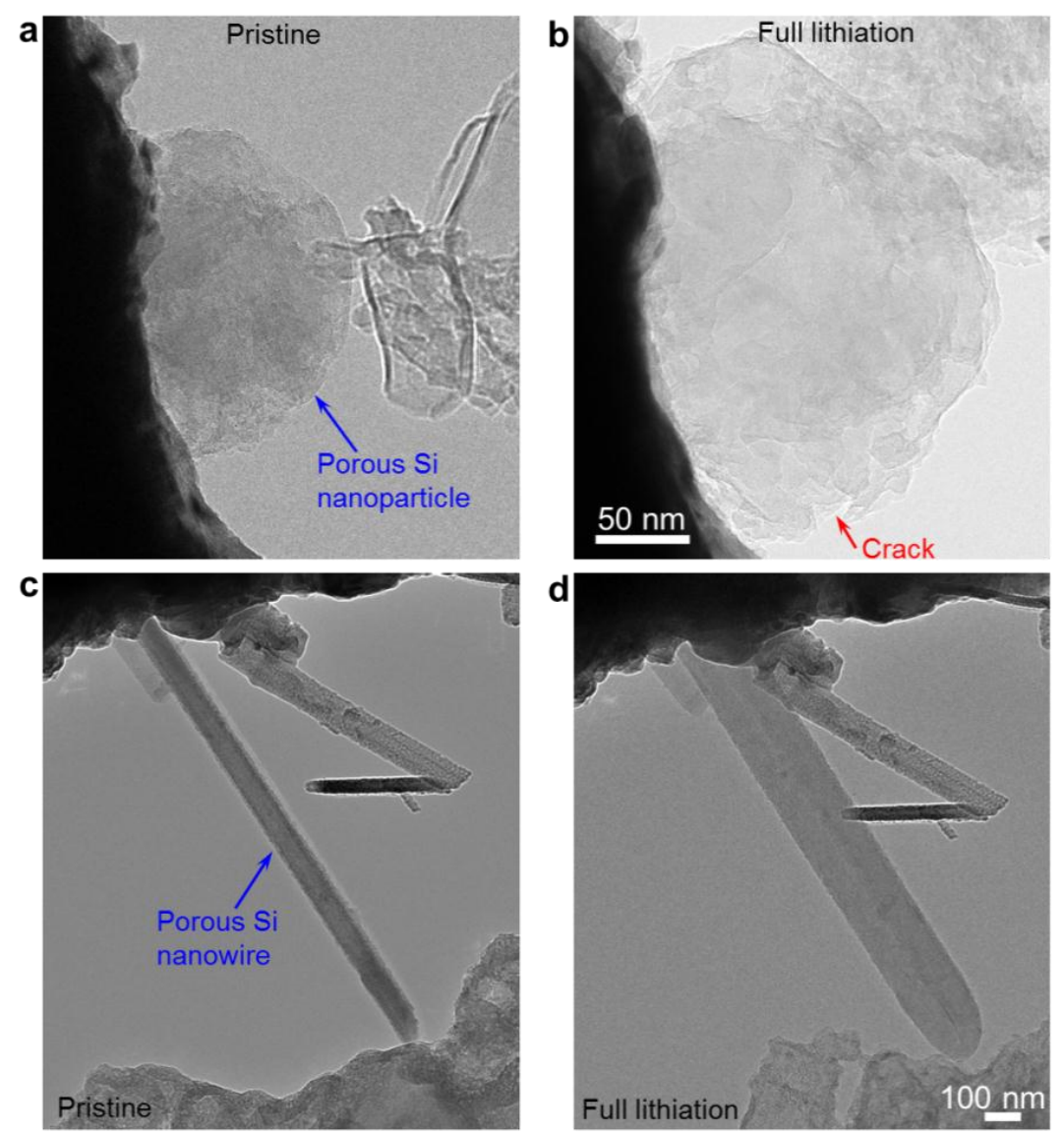

Figure S1 Lithiation behavior of porous Si nanoparticles and nanowires. Significant volume expansion occurred after the lithiation of porous $\mathrm{Si}$ nanoparticles (a-b) and nanowires (c-d). For porous Si nanoparticles and nanowires, the overall volume expansions after full lithiation are $\sim 280 \%$ and $\sim 320 \%$, respectively. It can be seen that the outward expansions of these porous anodes are still significant. We note that the volume expansion of the porous Si nanowire is higher than the theoretical value. This can be primarily attributed to the fact that the volume expansion of crystalline Si nanoparticles and nanowires cannot be precisely measured based on the $2 \mathrm{D}$ image due to the anisotropic expansion of crystal Si. Therefore, the introduction of a small porosity has a limited effect of alleviating the outward expansion. 


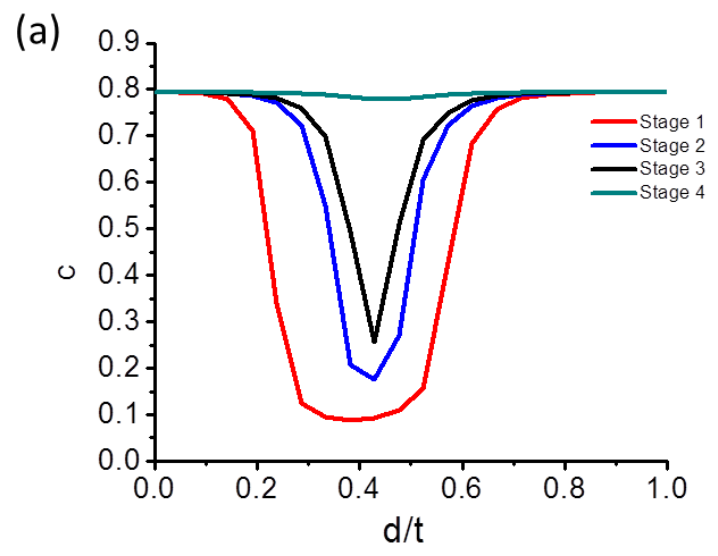

(b)

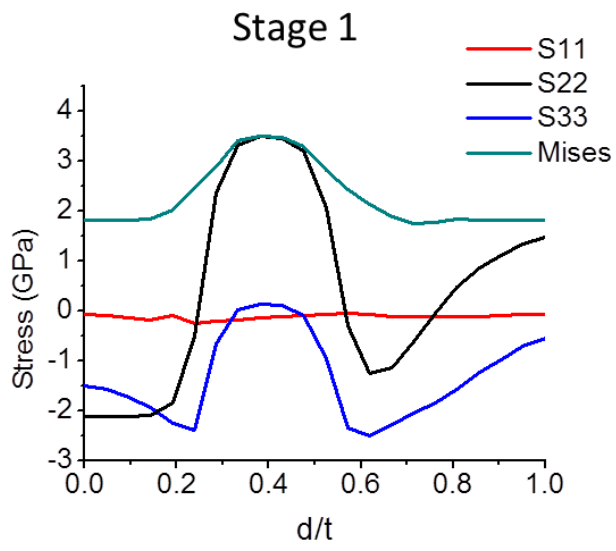

(d)

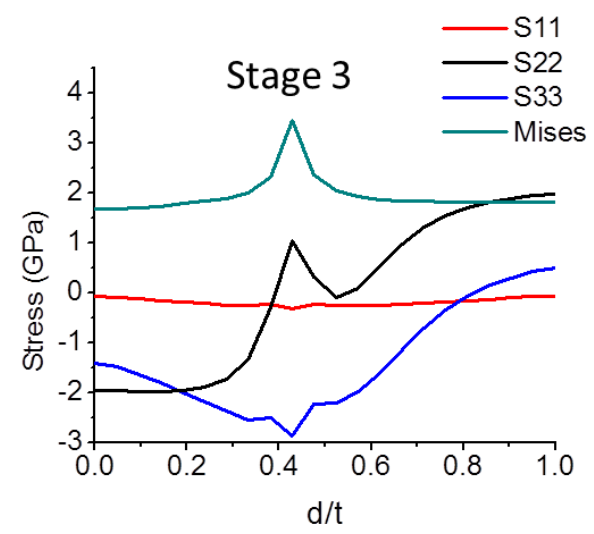

(c)

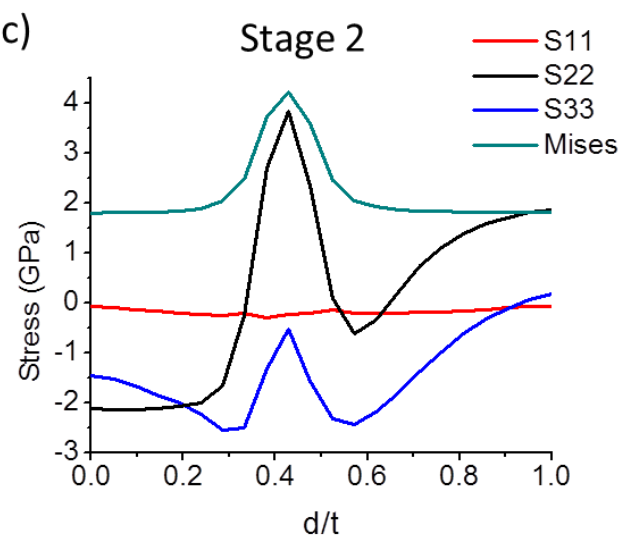

(e)

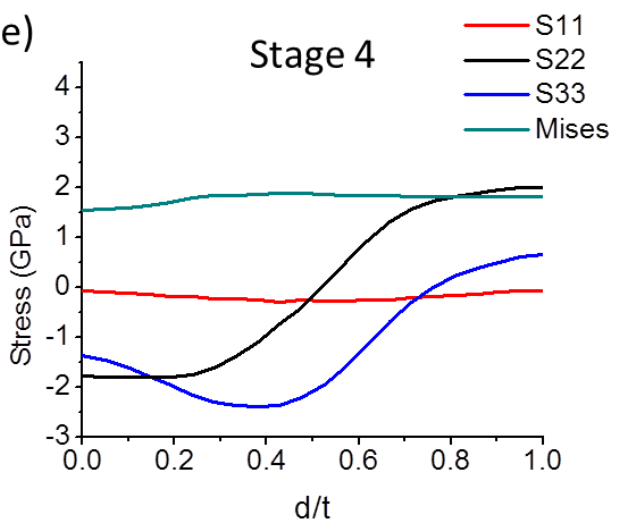

Figure S2 Chemomechanical simulation results of the sandwich two-phase lithiation in an $\boldsymbol{a}$-Si nanotube, after lithiation starts at the inner surface. (a) Radial distribution of the normalized $\mathrm{Li}$ concentration $\mathrm{c}$ (i.e., $\mathrm{Li}_{2.3} \mathrm{Si}$ corresponds to $\mathrm{c}=0.8$ ) at four characteristic stages. The symbol of $d$ denotes the distance from the inner surface of the nanotube, and $t$ is the thickness of the nanotube wall. (b-e) Corresponding radial distributions of stresses at four stages, S11 and S22 are respectively the radial and hoop stress in the cross section, and S33 is the axial stress in the nanotube. All the distributions are plotted on the undeformed configuration using ABAQUS, so as to facilitate comparison between different stages. 


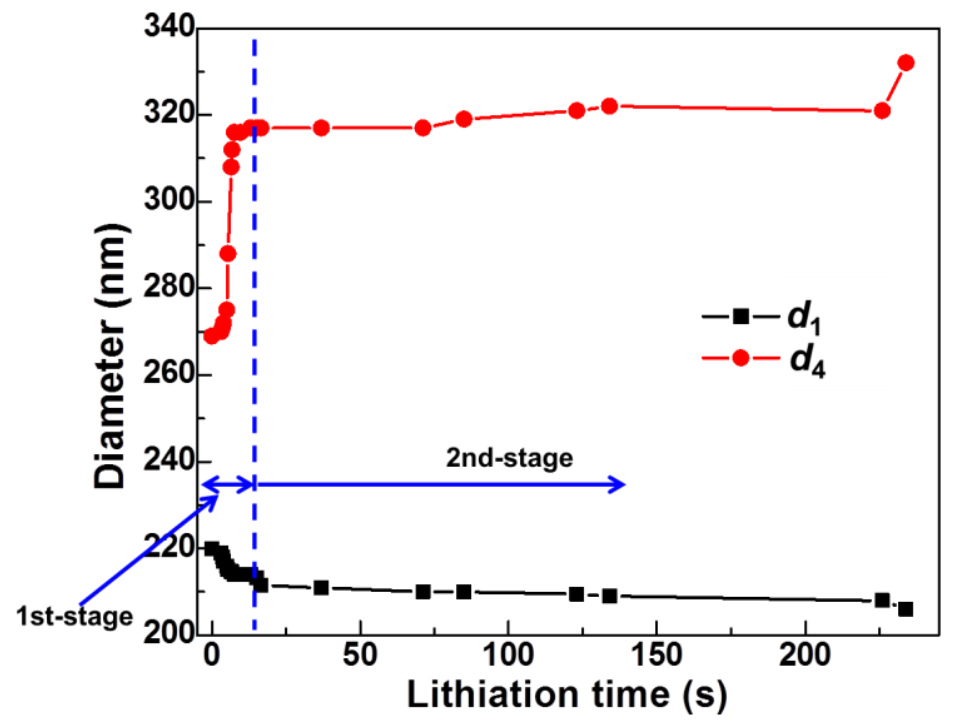

Figure $\mathrm{S3}$ Lithiation dynamics of a $3 \| 4 \mathrm{~nm} \mathrm{SiO} / \mathrm{Si} / \mathrm{SiO}{ }_{x}$ nanotube, showing a twostage lithiation process. The diameter-time curves were measured at a given position of the nanotube. The identification of different lithiation stages is the same as the method used in Figure 1.

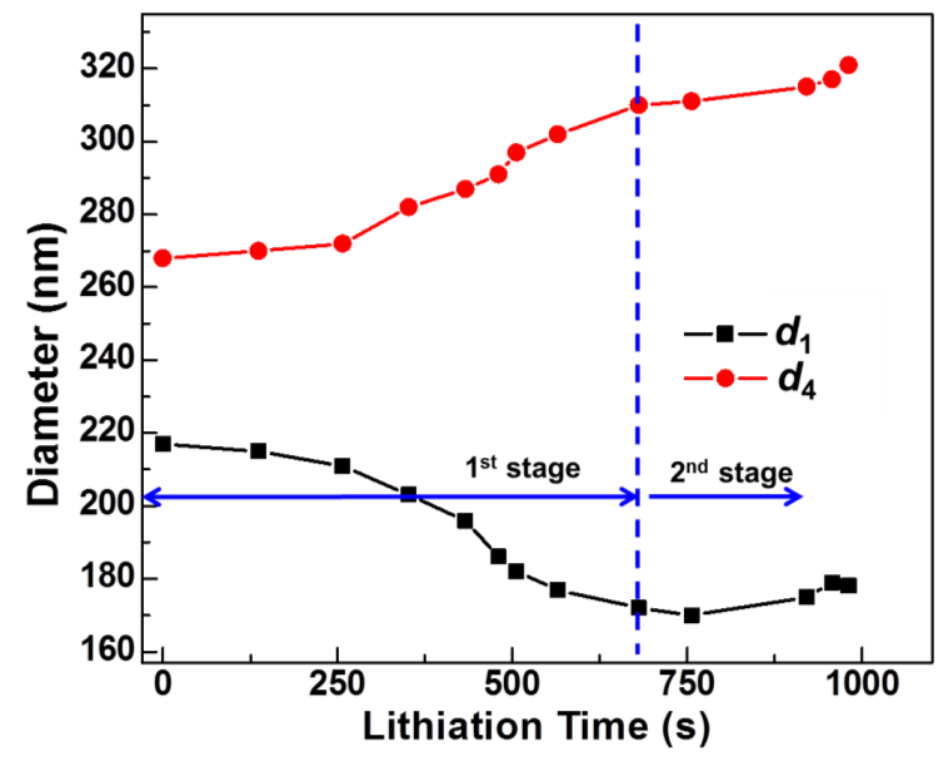

Figure $\mathrm{S} 4$ Lithiation dynamics of a $4 \| 6.5 \mathrm{~nm} \mathrm{SiO} / \mathrm{Si} / \mathrm{SiO}$ nanotube. The diametertime curves were measured at a given position of the nanotube. The identification of different lithiation stages is the same as the method used in Figure 1. 


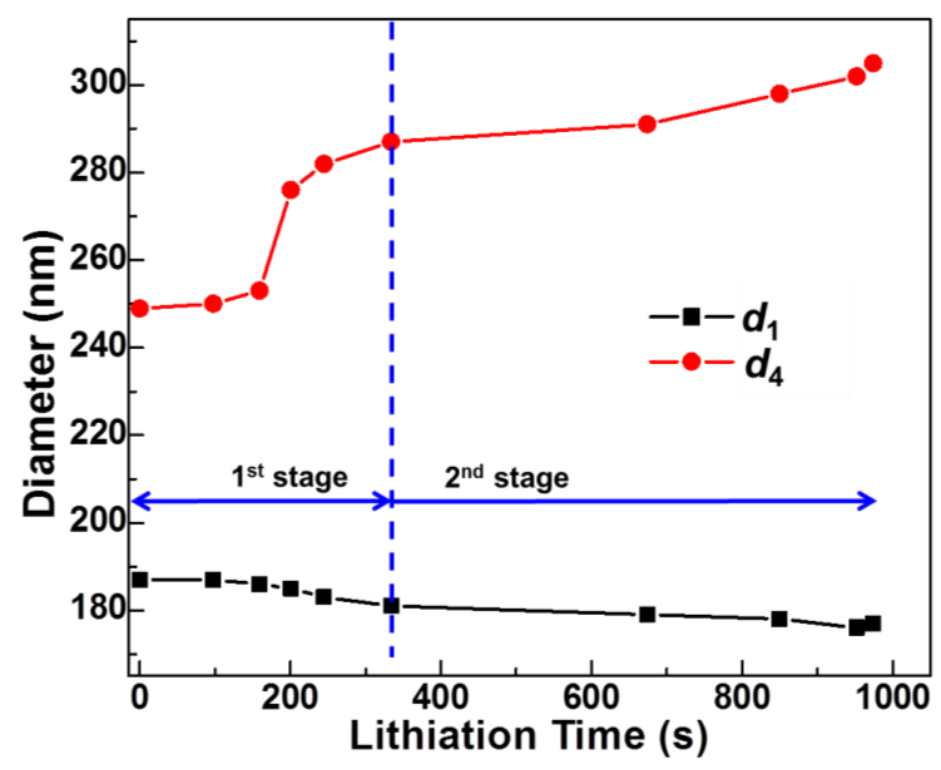

Figure $\mathrm{S5}$ Lithiation dynamics of a $6.5 \| 9 \mathrm{~nm} \mathrm{SiO} / \mathrm{Si} / \mathrm{SiO}$ nanotube. The diametertime curves were measured at a given position of the nanotube. The identification of different lithiation stages is the same as the method used in Figure 1. In the in situ TEM electrochemical testing, the lithiation dynamics can be affected by the experimental configuration and sample conditions. In Figure S3 and Figure S4, the lithiation experiments were conducted under a similar applied voltage of $-3 \mathrm{~V}$. Due to the poor conductivity of $\mathrm{SiO}_{x}$, a longer lithiation time was needed for the diffusion of lithium ions through a thicker surface oxide. This is likely the reason why the first stage lithiation in Figure S4 took much more time than that in Figure S3. However, in Figure S5, a much higher potential $(-20 \mathrm{~V})$ had to be applied to initialize the lithiation of $6.5 \| 9 \mathrm{~nm}$ $\mathrm{SiO}_{\mathrm{x}} / \mathrm{Si} / \mathrm{SiO}_{\mathrm{x}}$ nanotube. Once the $\mathrm{SiO}_{\mathrm{x}}$ coating was lithiated, the lithiation of $a$-Si can proceed very fast under the high potential applied.

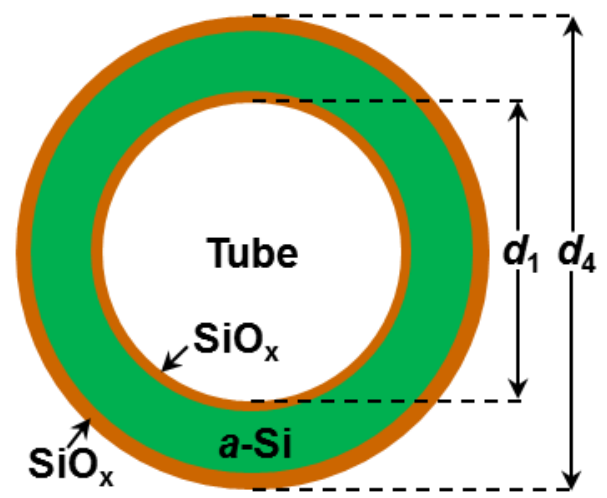

Figure S6 Schematic of the model representing the cross section of a $6.5 \| 9 \mathrm{~nm}$ $\mathrm{SiO}_{\mathrm{x}} / \mathrm{Si} / \mathrm{SiO}_{\mathrm{x}}$ nanotube. 

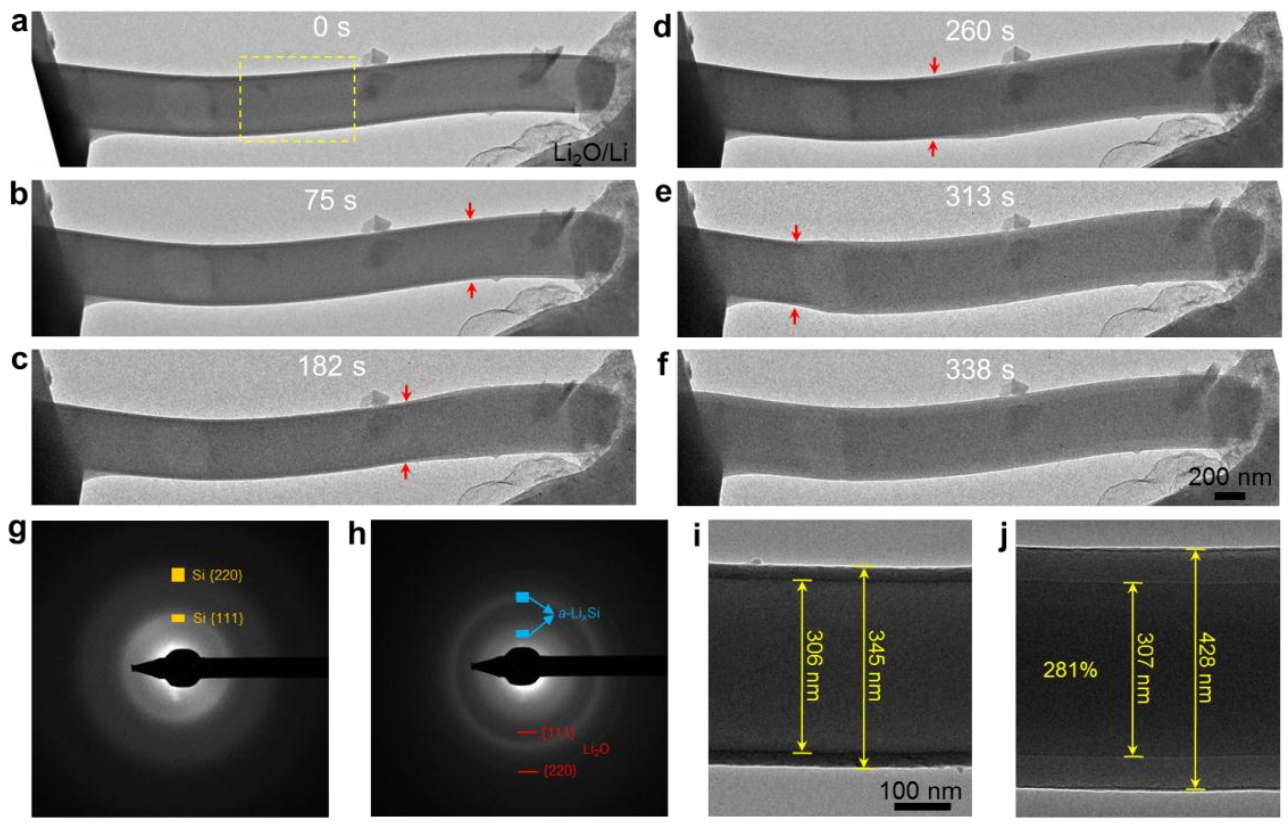

Figure S7. Lithiation process of an $\boldsymbol{a}$-Si nanotube at low-magnification. (a-f) Timelapse images of lithiation of the $a$-Si nanotube, showing the sandwich structure and twostage lithiation process. (g-h) Diffraction patterns of the nanotube before $(\mathrm{g})$ and after $(\mathrm{h})$ the full lithiation. (i-j) Diameters of the nanotube before (i) and after (j) the lithiation. It can be seen that the wall thickness and surface oxides cannot be clearly identified under low magnification. Since the main purpose of this work is to clearly understand the lithiation behavior and lithiation dynamics of $a$-Si nanotubes with different surface $\mathrm{SiO}_{\mathrm{x}}$ layers, we presented in the paper the real-time high-magnification images (with the nanometer resolution), by which the geometrical changes during lithiation can be precisely measured. 